\title{
An Internet-based Accounting Information Systems Project
}

Louise Miller, Robert Morris University, USA

\begin{abstract}
This paper describes a student project assignment used in an accounting information systems course. We are now truly immersed in the internet age, and while many required accounting information systems courses and textbooks introduce database design, accounting software development, cloud computing, and internet security, projects involving the actual integration of these topics with accounting concepts in a single comprehensive student project on the internet are uncommon. The project described gives students experience and knowledge of an internetbased accounting system using an online database. Although this project was used in an accounting information systems course, it could also be utilized in other courses such as introductory database management systems, advanced web design, financial technology systems, or introductory information technology. The project can be easily modified to align with the experience and sophistication of the students taking the course.
\end{abstract}

Keywords: Accounting Information Systems; Internet

\section{INTRODUCTION}

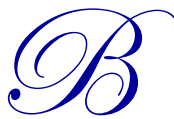

oth accounting educators and practitioners have historically agreed that Accounting Information Systems (AIS) courses should enable accounting students to become more proficient in using information systems (AAA 1987; AICPA 1996; Albrecht and Sack 2000; Hastings et al. 2003; International Federation of Accountants 2003). Also, technology in all forms, especially internet technology, is becoming integral and essential to accounting systems for both large and small businesses. The AICPA has defined one of the core competencies of students as being Technologically Adept, or "able to utilize and leverage technology in ways that add value to clients, customers and employers" (AICPA 1996). The word "technology" in an accounting setting has traditionally referred to students being adept with spreadsheets, accounting software packages such as QuickBooks, or database software such as Microsoft Access. But as Badua (2008) concludes, the scope of AIS is greater and more profound than the technical operations of accounting software. And we are now being rapidly immersed into internet technology at all levels and in all areas of accounting from financial reporting and XBRL to ecommerce systems, online ERP systems and other outsourced accounting-related services. Hot topics in accounting technology include Accounting and Business Intelligence updates with mobile apps for the iPhone and iPad, and cloud computing. An indication of the importance of the latter is a Bloomberg interview with Microsoft International President Jean-Phillipe Courtois, who indicated that Microsoft will spend 90 percent of its $\$ 9.6$ billion research and development budget on cloud strategy in 2011. (Eksin 2011). Cloud computing essentially refers to any services or applications that are hosted and accessed via the internet. Cloud computing allows small businesses to remotely manage and store information, and access customized software, services, or information from virtually any device with internet access. As cloud computing evolves, it is transforming accounting, moving accounting software to the cloud. The move to the cloud has the potential to increase collaboration and efficiency, and reduce costs for businesses, but it also presents challenges to AIS educators.

So the accounting profession is now truly in the internet age, and while many required accounting information systems courses and textbooks introduce database design, accounting software development, and internet security (Romney and Steinbart 2003; Kay and Ovlia 2012), projects involving the actual integration of these topics with accounting concepts in a hands-on student project on the internet are uncommon. This paper describes such a student project assignment used in an accounting information systems course. This project gives 
students actual "real world" experience and knowledge of an internet-based accounting system. Although this project was used in an accounting course, it could also be utilized in other courses such as introductory database management systems, advanced web design, financial technology systems, or introductory information technology. The project can be easily modified to align with the experience and sophistication of the students taking the course. The project described here was used in an introductory accounting information system class, taken primarily by sophomore and junior accounting and finance majors, so no prior knowledge of database systems or systems development was assumed or expected. The project also does not assume any prior knowledge of website design, query languages, or software coding. Adapting the project to upper-level courses for students with a greater knowledge of internet programming would be straightforward. This project can also be used as a class demonstration in cost accounting or managerial accounting courses, to illustrate real-world application of cost allocation, activity-based costing, and managerial decision-making strategies in an internet environment.

The fundamental idea of this project is to create a simple financial application for a healthcare organization in a website that uses a database that can be accessed via the internet. In other words, the user interface, the database and all software for the application reside in "the cloud". The instructor of the course initially sets up a webhosting account and registers an internet domain name. This does not require as large investment as it would have in the past, because virtually all web hosting companies provide all the services and tools required for a sophisticated internet site that is integrated with a database in their basic level web-hosting service. For the project described here, Yahoo Webhosting was used at a cost of only $\$ 9.95$ per month. This low cost includes unlimited space, multiple users with selective access levels, and multiple databases. The fundamental process for the instructor in this project is setting up the web hosting environment and then giving all students taking the course access as administrators to be allowed to create or modify an accounting information system. Administrator rights give students complete access to the database tools and admin areas to give them knowledge and experience with the management of an actual accounting software implementation on the internet. (Although the instructions and descriptions here are specific to Yahoo Webhosting, all web hosting services provide similar services and administrative access webpages.)

Most enterprise databases used in accounting information systems are built with sophisticated and powerful database software such as MS SQL Server or Oracle DBMS. These databases are uploaded to the web and software such as SQL, a query and data manipulation language, is used to access and modify the database. PHP is an HTMLembedded scripting language designed for web development to produce dynamic web pages. PHP code is embedded into the HTML source document and interpreted by a web server with a PHP processor module, which generates the web page document. PHP can be deployed on web servers on almost every operating system and platform free of charge. SQL statements to interact with the database are embedded into the PHP code, and that code displays the results on a webpage. PHP is installed on more than twenty million websites and one million web servers. Due to this popularity, virtually all web hosting services provide free tools for PHP coding and database interaction. The phpMyAdmin tool is a free open-source utility that is made available through the Yahoo Webhosting Control Panel. This tool handles the administration of MySQL over the Web. It can create and import databases, create and alter database tables and fields, and execute any SQL statement.

The student project described here should be used towards the end of an accounting information systems course (or information systems course), because it melds many subjects that would be covered in a typical course of this type. It integrates the topics (or has elements of) internet databases and database design, dynamic web pages, basic HTML commands, query languages and fundamental SQL statements, cloud computing and web hosting services, access control and cybersecurity, the PHP language, accounting system development, website administration functions and access logs, and systems design and documentation. If the students are also taking a cost accounting course, this project is a useful companion to topics in that course because it highlights the topics of activity-based cost allocation and cost analysis for pricing decisions.

Students are first asked to prepare a flowchart and dataflow diagram for depicting the overall process of the healthcare system described in the project. These system diagrams are an integral component of modern system documentation and have become increasingly important in response to heightened awareness surrounding compliance concerns and with legislation such as the Sarbanes-Oxley Act. Students can be guided to additional materials pertaining to system documentation (Lehman 2000) and advanced students can be asked to prepare other 
types of system diagrams. Additional materials may be suggested here because certain systems diagramming techniques used in practice are not well represented in curricula and texts (Bradford, et al. 2007).

\section{PROJECT SUMMARY DESCRIPTION}

The fictitious ACME Health Center is a chain of day surgery centers located across the United States. The student's job is to create (or modify) a simple website that implements the pricing information for a surgical procedure. Information about the surgical procedure is contained in a database that resides within the webhosting account that is set up for the course. All students have administrator access to the webhosting account, where they each create their own website and code for implementing their unique assignment. After implementing their website, each student uses the website to calculate the price of a surgical procedure. The student also must implement security measures to protect access to their particular webpages.

The fundamental steps for project setup by the instructor of the course prior to assigning the project to the students are:

1) Register a domain name for the course's website. (e.g. www.acmeheathcenterAISclass.com)

2) Sign up for the basic web hosting service with Yahoo Webhosting for this domain.

3) Using Yahoo's password manager, create a username and password for student access to the account. Grant administrator rights for this username so that all students in the class can access the web hosting control panels and functions.

4) In Yahoo Web Hosting, install the phpMyAdmin tool and use that tool to import spreadsheets containing the data for the initial Acme Health Center database. This installation is achieved with just a few steps and will require the assignment of a separate username and password to access the phpMyAdmin website.

5) Go to the filemanager and create a sample directory to store the webpages (code) for the ACME Health Center website.

In a pre-assignment classroom demonstration, the instructor enters the Yahoo Webhosting administrator panel for the course website. (Any modern university classroom provides internet access.) The instructor demonstrates how to login to the website control area and also how to enter the phpMyAdmin website, which allows viewing of the actual databases that reside in the website. The phpMyAdmin website also has many other functions available related to the databases, such as inserting or deleting records, assigning primary keys, sorting, or changing the actual structure of the database design. This classroom demonstration can reinforce many topics that have been covered in the course up to this point such as database design, functionality provided by web hosting services and databases in cloud computing. A good example of this functionality is website access logs, which are discussed in many accounting information systems textbooks (Romney and Steinbart 2008). Access logs provide information about visitors to websites, and are a useful tool in cybercrime investigation (hacker identification) as well as in gathering information about website popularity and demographics of visitors to business websites. The access logs contain information about IP addresses, webpages visited, access times, and operating systems of visiting computers. See Figure 1 for an example of the entries in a $\log$ file in Yahoo Webhosting that can be displayed and discussed with the students.

The instructor can display this current real-time log file for the course website and use an IP address information site such as cqcounter.com to reveal location information about visitors who have actually entered the website during the class just minutes before clicking on the access log. See Figure 2 for an example of the output of cqcounter.com for an IP address contained in the log shown in Figure 1. This shows the visitor to the website used an Internet Service Provider (ISP) located in Paris, France. 
Figure 1

Sample Access Log Entries in Yahoo Webhosting

$82.123 .207 .194-[21 /$ Aug/2011:13:11:43 -0700] "GET

/ACMEHealthCenterWebsite/ProcedurePricingFormHTML.htm HTTP/1.1" 2001442

"http://us.1.p8.webhosting.yahoo.com/filemanager/advancededitor" "Mozilla/4.0

(compatible; MSIE 8.0; Windows NT 6.1; Trident/4.0; SLCC2; .NET CLR 2.0.50727; .NET

CLR 3.5.30729; .NET CLR 3.0.30729; Media Center PC 6.0; InfoPath.3; .NET4.0C;

BRI/2) " "collegiumverita.com"

82.123.207.194 - [21/Aug/2011:13:12:06 -0700] "POST

/ACMEHealthCenterWebsite/ProcedurePriceCalcResults.php HTTP/1.1" 200254

"http://collegiumverita.com/ACMEHeal thCenterWebsite/ProcedurePricingFormHTML.htm"

"Mozilla/4.0 (compatible; MSIE 8.0; Windows NT 6.1; Trident/4.0; SLCC2; .NET CLR

2.0.50727; .NET CLR 3.5.30729; .NET CLR 3.0.30729; Media Center PC 6.0; InfoPath.3;

.NET4.OC; BRI/2)" "collegiumverita.com"

82.123.207.194 - [21/Aug/2011:13:12:11 -0700] "POST

/ACMEHealthCenterWebsite/ProcedurePriceCalcResults.php HTTP/1.1" 200254

"http://collegiumverita.com/ACMEHealthCenterWebsite/ProcedurePricingFormHTML.htm"

"Mozilla/4.0 (compatible; MSIE 8.0; Windows NT 6.1; Trident/4.0; SLCC2; .NET CLR

2.0.50727; .NET CLR 3.5.30729; .NET CLR 3.0.30729; Media Center PC 6.0; InfoPath.3;

.NET4.OC; BRI/2)" "collegiumverita.com"

Figure 2

Example of a Website that Analyzes IP Addresses

Whois
IP Address / Domain Name Lookup :-
\begin{tabular}{|l|l|l|}
\hline Site Info new & Who Is Trace Route Link Popularity RBL Check What's My IP? Web Search 口 \\
\hline Enter Domain Name or IP Address: & Whois \\
\hline 82.123 .207 .194 & Site Info \\
\hline
\end{tabular}

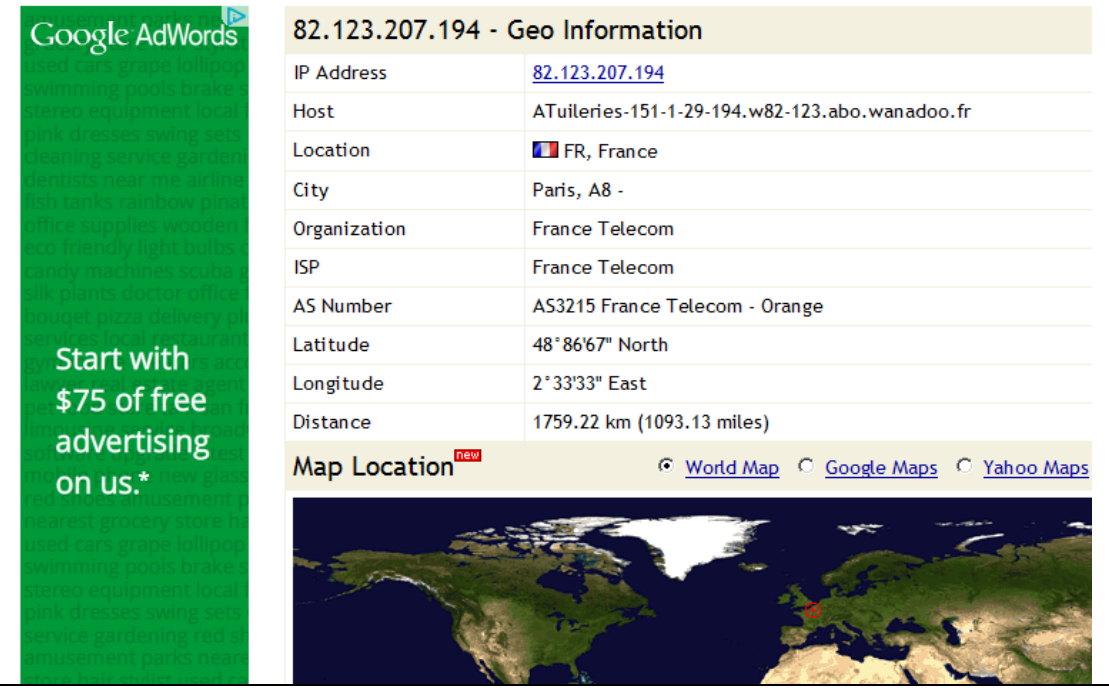

Following this classroom demonstration, the students are assigned a multi-part project, which can be submitted over several weeks. The parts are described in the following section.

\section{STUDENT PROJECT ASSIGNMENT IN EIGHT PARTS}

This is a condensed list. The actual assignment will contain additional specific information such as URL's, usernames, and passwords. (The full list is available from the author.) 
1) Students are asked to prepare a flowchart and dataflow diagram for depicting the overall process of the manager at ACME Health center using the site to calculate the price charged for a surgical procedure. Students are instructed as follows: "Assume that the manager must login to gain access to the site, and then calculates the price of a procedure for a patient. After the patient has undergone the procedure, a counter is updated in the procedure table for the number of times the procedure is performed. Patient information is maintained in the website, and updated as needed. Inventory information for medical devices is also maintained. In your diagrams include any data flows to/from the database utilized by the website."

2) HIPAA (Health Insurance Portability and Accountability Act) code sets for diagnosis and procedures are used in health care transactions. Students are asked to develop and describe a similar coding scheme for their ACME Health Center procedures. This part of the project facilitates the learning of coding schemes used in accounting information systems.

3) Students are given these instructions:

Go to yahoo.com and login with the student username and password given to you by your instructor. Go to the web hosting control panel and browse around the site and see the functions available there. List the locations of the real-time usage graphs and access logs, and describe the basic functionality provided for the website administrator. The file manager is where files and folders are manipulated, stored, and organized. One folder contains the database code files (the code to run the database functions) and files in this directory are not to be used in this project. Create your own student folder (subdirectory) in the main folder of the file manager. The simple website for the ACME Health center consists of three webpages: a main page, a procedure pricing page with an input form, and a procedure pricing results page. Copy these three files (supplied by the instructor) into your personal subdirectory:

- ACMEHealthCenterMainPage.html

- ProcedurePricingFormHTML.htm

- $\quad$ ProcedurePriceCalcResults.php

The students will now have their own ACME Health Center website. For advanced courses, in which students are familiar with HTML, the main page creation can be a requirement, or advanced enhancements can be required here.

4) Students are given these instructions:

In the web hosting account, a mysql database has been set up called ACMEHealthCenter. This is the database that the code in the ACME Health Center website will interact with when managers use the website. In the database there this table has been uploaded: ProcedureList. To look at the database and the tables in it go to the website link given to you by your instructor and enter with the student username and password. Figure 3 is an example of the display you will see when you browse the ProcedureList table. Create a new sample patient table in Excel, with personal information for 20 patients, including patient number and type of insurance coverage. Import the patient spreadsheet into the database into a table called YourNamePatientList. Add a primary key to your table.

5) In this project, the managers of ACME Health Center want to assign a price to a procedure, and using prior data they have devised a formula similar to this:

$$
\begin{aligned}
& \text { Cost }=\$ 200 * \text { OperatingRoomType }+\$ 10 * \text { PreOpMinutes }+\$ 50 * \text { ProcedureMinutes }+\$ 20 \\
& * \text { PostOpMinutes }+\$ 50 * \text { Number of Nurses }+\$ 80 * \text { DeviceLevel }+\$ 90 * \text { AnesthesiaLevel } \\
& + \text { OfficeOverhead }
\end{aligned}
$$

Each of the independent variables in this formula except OfficeOverhead are fields in the database table for procedures. The main webpage (ACMEHealthCenterMainPage.html) shown in Figure 4 contains a link to the procedure pricing page. Yahoo Webhosting (and any other webhosting service) provides a simple wizard to quickly build a basic webpage such as this. After entering information into the form for the procedure pricing request, shown in Figure 5, the "Calculate Procedure Price" button is pressed and the procedure pricing results page is displayed. The code modules ProcedurePricingFormHTML.htm and ProcedurePriceCalcResults.php, shown in Figure 6 and Figure 7, handle form display and the surgery procedure price calculation that is done on the website. 
Figure 3

Display of the Procedure List Table in the phpMyAdmin utility

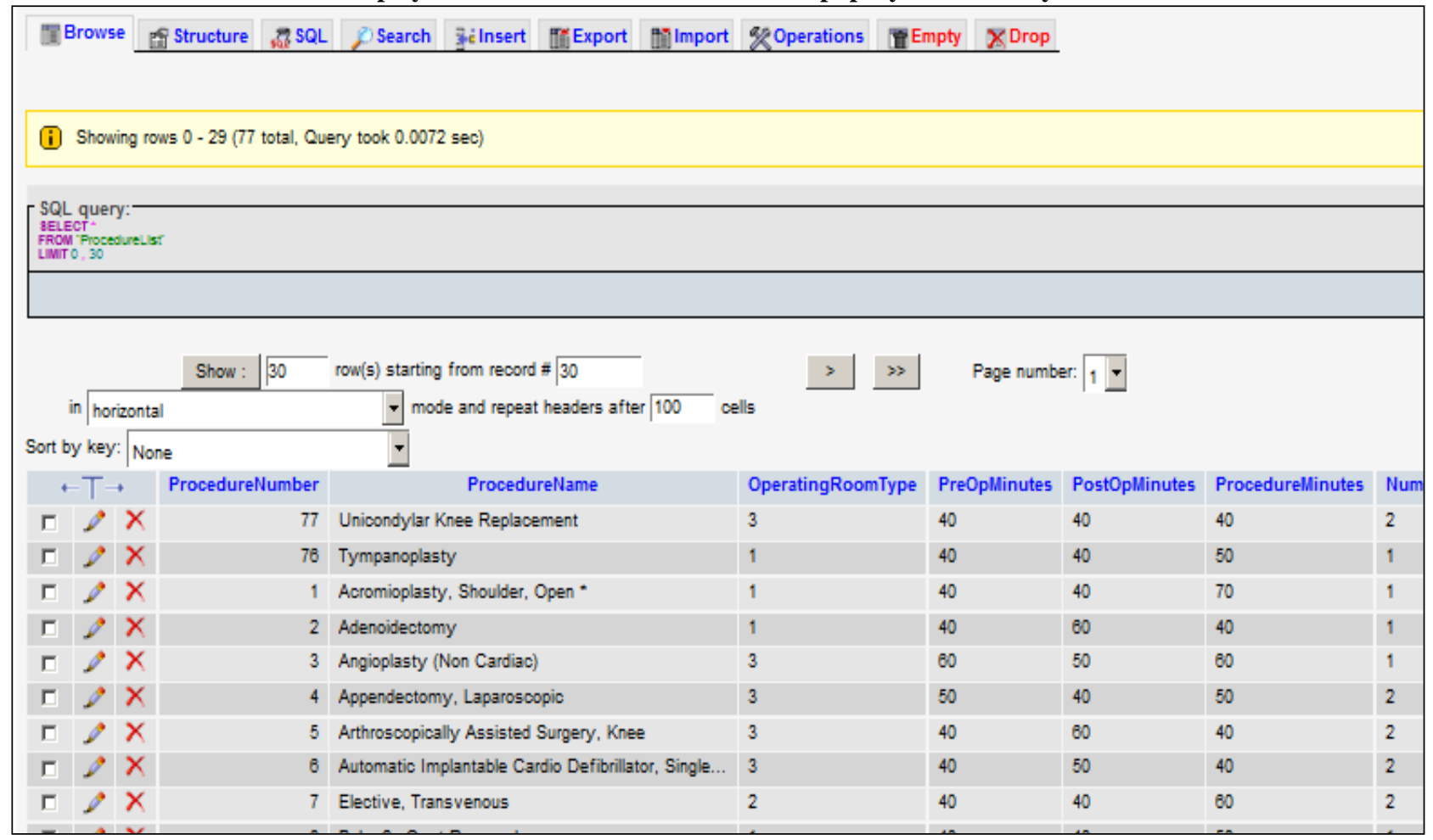

Figure 4

The main webpage

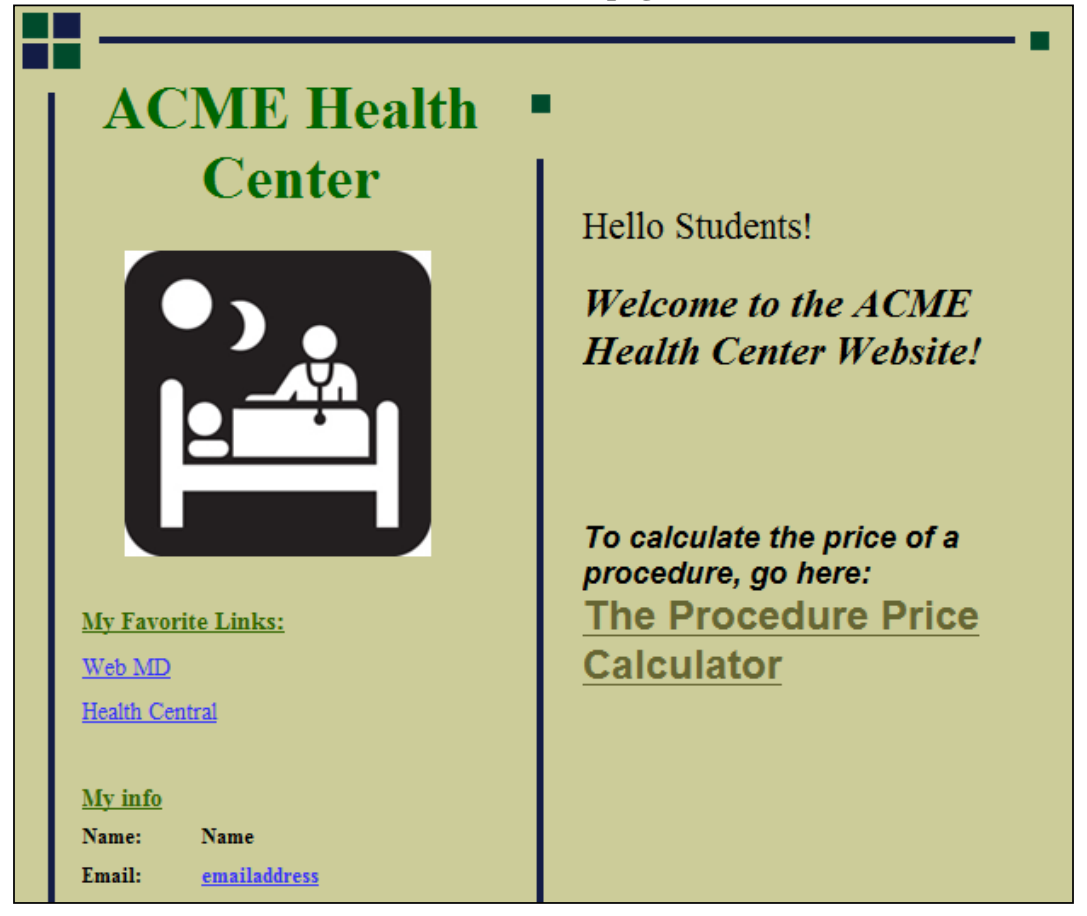


Figure 5

Procedure Pricing Request Form

Medical Procedure Pric
Choose Markup Percentage:
Choose Overhead Category:
Enter Procedure Name:
Tympanoplasty
Calculate Procedure Price

Figure 6

HTML Code for the Procedure Pricing Request Form

$<$ title $>$ Medical Procedure Price Calculation</title>

$<\mathrm{h} 1>$ Select Procedure Detail: $</ \mathrm{h} 1>$

<form action="http://louiseannette.com/ACMEACC2080Project/ProcedurePriceCalculationResults.php" method="post">

Choose Markup Percentage: $<$ br $/>$

$<$ select name="MarkupPercentage" $>$

<option value $=40 ">40 \%<$ /option $>$

$<$ option value $=" 50 ">50 \%<$ /option $>$

$<$ option value $=" 60 ">60 \%</$ option $>$

$</$ select $>$

$\langle$ br $/>\langle$ br $/>$

Choose Insurance: $\langle\mathrm{br} /\rangle$

$<$ select name="AnesthesiaLevel" >

<option value $=" 1 ">$ Level $1<$ option>

$<$ option value $=" 2 ">$ Level $2<$ option $>$

$<$ option value $=" 3 ">$ Level $3<$ option $>$

$</$ select $>$

$\langle\mathrm{br} /\rangle\langle\mathrm{br} /\rangle$

Enter Procedure Name: $\langle$ br $/>$

$<$ input name="searchProcedureName" type=""text" size="30"/>

$\langle$ br $/>$

$<$ input type="submit" name="submit" value="Calculate Procedure Price"/>

$</$ form $>$

Figure 7

Code for Procedure Price Calculation and Display of the Results Webpage

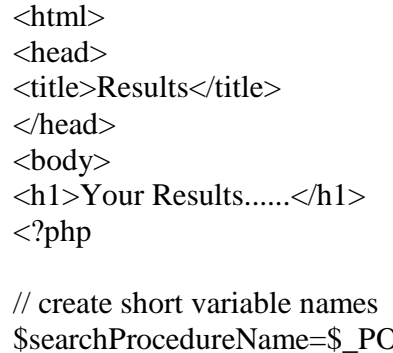

\$searchProcedureName=\$_POST['searchProcedureName'];

//CONNECT TO MYSQL

\$mysql_access = mysql_connect('mysql', 'pillow_lady', 'abcabc12');

C) 2012 The Clute Institute http://www.cluteinstitute.com/ 


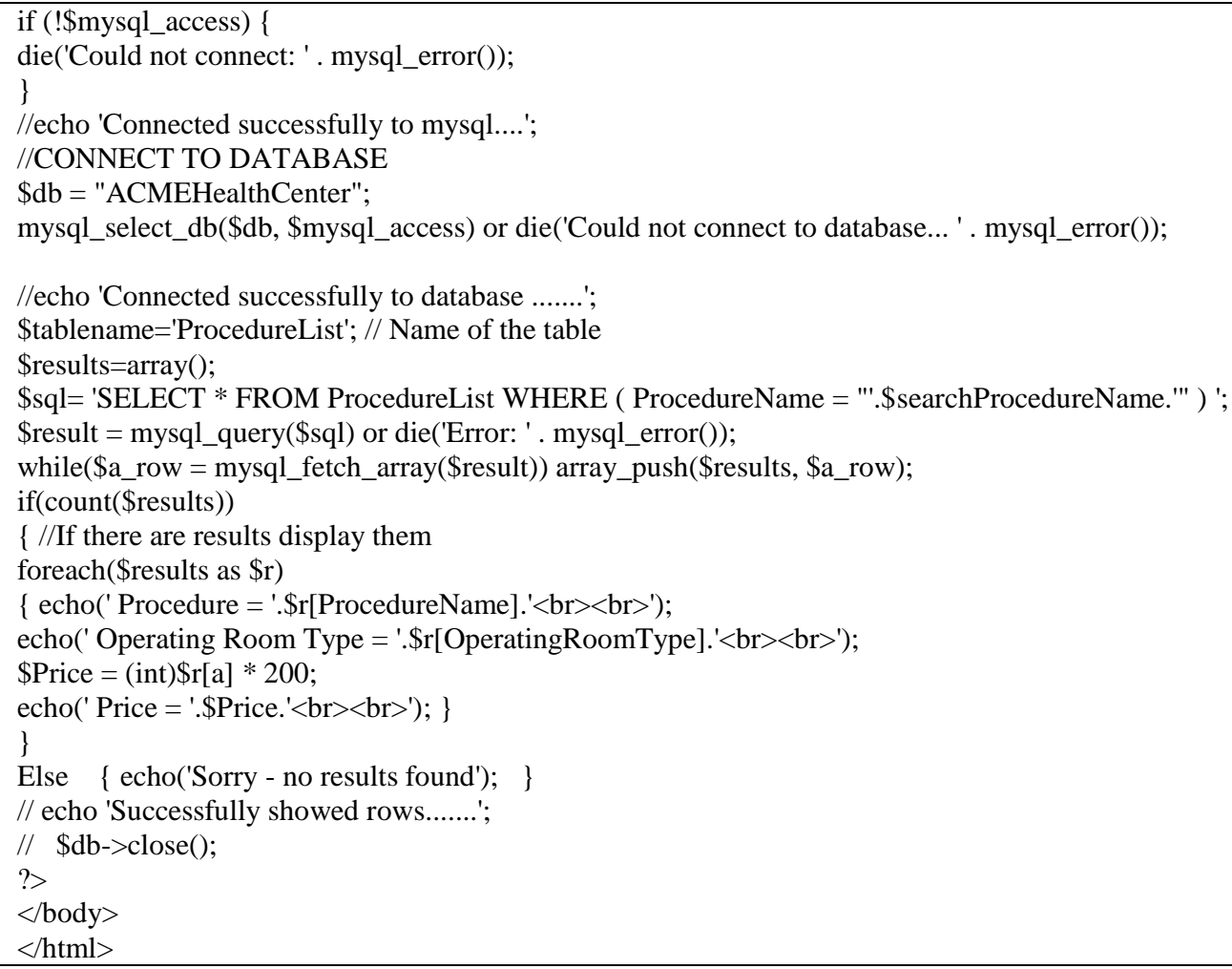

The code in ProcedurePriceCalcResults.php implements the pricing algorithm and the students are asked to modify this algorithm in the code. The code is pulling the information from the database table and using that to calculate the price. More advanced students in information technology courses can be asked to create the procedure pricing results page code themselves or implement additional functionality, rather than modifying existing code. This part of the project facilitates understanding of the basics of query languages and fundamental SQL statements, and their relationship to databases.

For student assessment of this portion of the project, each student can be assigned different numbers for various pop-down menu values, given different algorithms, and asked to modify the code for their unique assignment. The students are then asked to use their website to calculate a price for a medical procedure. Assigning unique tasks for each student in the class may be time-consuming for the instructor, but assures individual work on the project. Grading in large classes can be facilitated by creating a spreadsheet where each student is assigned a student number and then the pricing algorithm is set as a function of their student number. If the correct code modifications have been done then the answer presented by the student website should match the answer given in the spreadsheet.

6) Students are given these instructions:

"Create a new subdirectory in your folder at the Yahoo.com site called "PrivateInfo". Add password protection to your PrivateInfo folder. Create a medical devices list in a spreadsheet and convert this to an HTML file. Upload that file into your PrivateInfo folder at the yahoo.com site. Add a link on your main page (of ACME Health Center) to your medical devices file. Send the username and password for your folder to the instructor to allow entry to your private folder to view the medical devices list."

This part of the project facilitates learning the topics of access control and cybersecurity related to accounting information systems. 
7) Students are given these instructions:

Analyze the risks and security needs for ACME Health Center. The threats to the security of protected health information have been identified as:

Threat 1: a cleaning crew that arrives during the night might gain access to records stored on computers.

Threat 2: insurance claims are transmitted unencrypted across the internet from the main office to a clearinghouse.

Threat 3: there are no data backup systems or required data backup procedures.

Estimate the risk severity of these threats and describe possible remedial actions. Describe which actions you think would be most cost effective. Write a memo to the company manager describing your internal control recommendations.

This part of the project facilitates learning the topics of internal control, threats and countermeasures, and cybersecurity related to accounting information systems.

8) Students are given these instructions:

Summarize the capabilities of the ACME Health Center information system you have created during this course, explaining in detail the interactions and functions of the database located in "the cloud". Also explain what capabilities would need to be added to make this a complete pricing system, such as forms to enter new records into the database and procedures and functions for generating pricing reports for management. Create or update your system documentation to reflect the additions.

\section{CONCLUSION}

This paper describes a comprehensive student project on the internet used in an accounting information systems course. The project described is an accounting system using an online database which be easily modified to align with the experience and sophistication of the students. The project is a combination of class demonstration and student assignment that integrates the topics of internet databases, query languages, cloud computing and web hosting services, access control and cybersecurity, and systems design and documentation. This project could also be used in managerial or cost accounting courses because it highlights the topics of activity-based cost allocation and cost analysis for pricing decisions.

The specific learning objectives (functional competencies) of this project are for students to:

(1) Understand system diagramming methods.

(2) Understand coding schemes.

(3) Gain an understanding of the fundamentals of database design and implementation on the internet.

(4) Understand the basics of query languages and fundamental SQL statements, and their relationship to databases, cloud computing and web hosting services.

(5) Understand the fundamentals of risk assessment, internal controls, and access control for internet-based accounting systems.

For the first and second objectives, students analyze the AIS problem presented, create a system diagram for the process and create a coding scheme for medical procedures. For the third and fourth objectives, students are tasked with modifying an actual online AIS (website) and database for a health care center and are asked to perform administrative functions for the health care center website. Finally, for the fifth objective, students perform a basic risk assessment to identify key internal controls, and implement a file access control that may mitigate the health care center's risks. 
By mapping the project activities to functional competencies and directly measuring multiple outcomes in these activities, these competencies can be assessed in the course as prescribed in the accounting department's assessment plan.

\section{AUTHOR INFORMATION}

Dr. Louise Miller has a Ph.D. in Management Science, an MBA and a BS degree in Electrical Engineering. Dr. Miller has over nine years of experience in higher education and in addition, she spent 20 years as a software engineer. Her research interests include accounting information systems and the integration of technology into accounting education. Dr. Miller also enjoys painting and web site design and programming. She teaches for Robert Morris University in Moon Township, Pennsylvania. E-mail: millerl@rmu.edu

\section{REFERENCES}

1. Albrecht, W. S., and R. J. Sack. 2000. Accounting education: Charting the course through a perilous future. Accounting Education Series. Vol. 16. Sarasota, FL: American Accounting Association.

2. American Accounting Association (AAA). 1986. Bedford Committee-Committee on the Future Structure, Content, and Scope of Accounting Education. Future accounting education: Preparing for the expanding profession. Issues in Accounting Education 1 (1): 168-195.

3. American Institute of Certified Public Accountants (AICPA). 1996. Information Technology Competencies in the Accounting Profession: AICPA Implementation Strategies for IFAC International Education Guideline No. 11: Implications for Education and Practice. New York, NY: AICPA.

4. 2006. The AICPA Core Competency Framework. Retrieved March 21, 2012, from: http://www.aicpa.org/interestareas/accountingeducation/resources/pages/corecompetency.aspx

5. Bradford, M., S. B. Richtermeyer, and D. Roberts. 2007. System Diagramming Techniques: An Analysis of Methods Used in Accounting Education and Practice, Journal of Information Systems, 21 (1): 173-212.

6. Kay, D., and A. Ovlia. 2012. Accounting Information Systems. New York, NY: Prentice Hall.

7. Romney, M., and P. Steinbart. 2008. Accounting Information Systems. 11th edition. Upper Saddle River, NJ: Prentice Hall.

8. Eksin, A. (2011, April 7). Microsoft Says to Spend 90\% of R\&D on Cloud Strategy. Bloomberg Businessweek. Retrieved August 11, 2011, from http://www.businessweek.com/news/2011-0407/microsoft-says-to-spend-90-of-r-d-on-cloud-strategy.html

9. Hastings, C. I., P. M. J. Reckers, and L. Solomon. 2003. The State of Accounting Curriculum: Where It Is and Where It Needs To Be. Proceedings of the Accounting Information Systems Research Symposium, Scottsdale, AZ.

10. International Federation of Accountants (IFAC). 2003. Information technology in the accounting curriculum: International education guideline No. 11. New York, NY: IFAC.

11. Lehman, M. 2000. Flowcharting made simple. Journal of Accountancy 190 (4): 77-85. 